\title{
Online-Zeitschrift, Altertumskunde und Nachwuchswissenschaft. Ein Erfahrungsbericht anlässlich der 10. Ausgabe von FeRA
}

\author{
Stefan Krmnicek - Peter Probst
}

Seit März 2006 ist die Zeitschrift „Frankfurter elektronische Rundschau zur Altertumskunde“ online unter www.fera-journal.eu abrufbar. Nach nunmehr gut vier Jahren und mit dem Erscheinen der zehnten Ausgabe sehen die Herausgeber die Möglichkeit gegeben, mit Blick auf das bisher Geleistete ein erstes Fazit zu ziehen und auf der Grundlage ihrer Erfahrungen die gegenwärtigen Rahmenbedingungen und Perspektiven des Publizierens elektronischer Zeitschriften in der Altertumskunde zu diskutieren. $^{1}$

Die Verfasser dieses Beitrages setzten sich mit dem Projekt „Frankfurter elektronische Rundschau zur Altertumskunde“ zum Ziel, eine international ausgerichtete altertumswissenschaftliche online-Fachzeitschrift von und für qualifizierte junge Nachwuchswissenschaftler zu begründen. Die Mehrzahl der zu diesem Zeitpunkt online verfügbaren Fachzeitschriften bestand aus digitalen, großteils nicht kostenfrei zugänglichen Parallelausgaben der herkömmlich erschienenen PrintVersionen. Insgesamt existierten nur wenige Fachzeitschriften auf dem Gebiet der klassischen Altertumskunde, die ausschließlich in digitaler Form erschienen. ${ }^{2}$ Zudem war in der Gruppe dieser Publikationen meist die inhaltliche Ausrichtung auf einen Teilbereich der klassischen Altertumskunde festzustellen. ${ }^{3}$ Ebenso gab es, von Ausnahmen in Großbritannien abgesehen ${ }^{4}$, keine frei verfügbaren Fachzeitschriften, die sich explizit als ein für den wissenschaftlichen Nachwuchs konzipiertes Projekt mit breit angelegtem thematischem Horizont verstanden. Im Gegensatz zu den meisten anderen online-Zeitschriften mit bloß einer Ausgabe pro Jahr oder unregelmäßigem Erscheinungsintervall wurde bei dem Frankfurter Projekt das periodische Erscheinen von drei Ausgaben pro Jahr vorgesehen, um für eine rasche Kommunikation der Forschungsergebnisse zu sorgen. Somit verbindet die „Frankfurter elektronische Rundschau zur Altertumskunde“ wesentliche Elemente bereits bestehender Online-Zeitschriften aus dem Bereich der Altertumswissenschaft und sticht aufgrund der Kombination dieser Elemente (speziell von und für Nachwuchswissenschaftler; lizenz- und kostenfrei; inhaltlich die gesamte Breite der klassischen Altertumskunde umfassend) gleichwohl als eine Besonderheit unter diesen hervor.

Bislang erschienen zehn Ausgaben, die insgesamt 36 Beiträge zu verschiedenen Themen der Altertumskunde von unterschiedlichster geographischer und chronologischer Streuung umfassen. Das thematische Spektrum der Beiträge in

\footnotetext{
${ }^{1}$ Für grundsätzliche Beobachtungen zum elektronischen Publizieren in der Geisteswissenschaft vgl. Leiß 2006.

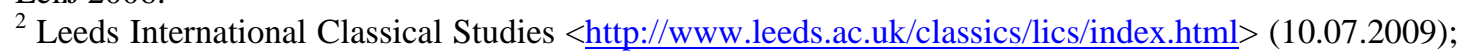
zu der Ausrichtung dieser Zeitschrift vgl. Heath 2002. Dagegen sind Online-Rezensionsjournale weitaus häufiger anzutreffen und haben sich, wie etwa BrynMawr Classical Review $<\underline{\text { http://bmcr.brynmawr.edu/> }>\quad \text { (10.07.2009), }}$ plekos $\quad$ http://www.plekos.unimuenchen.de/startseite.html $>\quad$ (10.07.2009) oder scholia <http://www.und.ac.za/und/classics/schrev/scholrev.html $>$ (10.07.2009), mittlerweile etabliert.

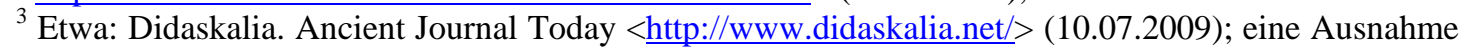
bildet: Forum Archaeologiae. Zeitschrift für klassische Archäologie < http://farch.net/> (10.07.2009).

${ }^{4}$ In der Konzeption am ähnlichsten: Digressus. The Internet Journal for the Classical World

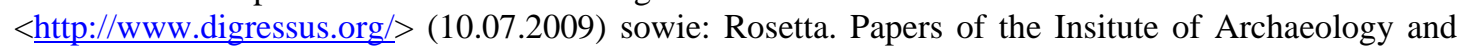

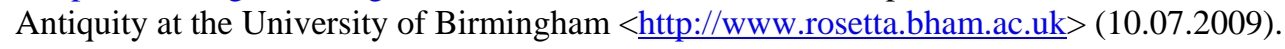


deutscher, englischer und italienischer Sprache reicht dabei von Untersuchungen zur materiellen Kultur bis hin $\mathrm{zu}$ schriftquellenorientierten althistorischen und philologischen Studien; eine Konzentration auf bestimmte Epochen der Antike oder bestimmte Regionen der Mittelmeerwelt lässt sich nicht ausmachen. Der Kreis der Autoren bestand fast ausschließlich aus jungen Nachwuchswissenschaftlern, die zum Zeitpunkt des Einreichens ihrer Manuskripte an der Dissertation schrieben oder diese vor Kurzem erfolgreich abgeschlossen hatten. Damit war das Vorhaben, den wissenschaftlichen Nachwuchs als primäre Zielgruppe anzusprechen, zumindest für die Autorenschaft erreicht.

Eine einfache Recherche im Internet dokumentiert die bisherige Außenwirkung ${ }^{5}$ des Projektes: die Suche nach der genauen Wortgruppe „Frankfurter elektronische Rundschau zur Altertumskunde“ (in Anführungszeichen) liefert bei der Internetsuchmaschine google ${ }^{\mathrm{TM}}$ über 150 Ergebnisse. Davon entfällt die Mehrzahl der gefundenen Seiten auf Internetpräsenzen aus dem Bildungs- und Wissenschaftsbereich (Universitätsbibliotheken, elektronische Zeitschriftenbibliotheken und -datenbanken, Open-Access-Initiativen u. ä.), bei denen die Adresse der „Frankfurter elektronischen Rundschau zur Altertumskunde“ neben anderen elektronischen Fachzeitschriften indiziert und per Link ein direkter Zugriff auf die Website www.fera-journal.eu möglich ist. Etwa $10 \%$ der gefundenen Seiten verdeutlichen die aktive fachliche Rezeption des Projektes: Artikel der „Frankfurter elektronischen Rundschau zur Altertumskunde“ werden sowohl in online verfügbaren Publikationen (digitale Zeitschriften, Lehrveranstaltungsmaterialen, u. ä.) als auch in sonstigen Facheinträgen (fachspezifische Websites, Wikis, u. ä) zitiert. Dies betrifft sowohl die Wahrnehmung des Projektes als solches im Rahmen einer Untersuchung des wissenschaftlichen Publizierens archäologischer Forschungsergebnisse im Internet als auch die Rezeption einzelner Beiträge. ${ }^{6}$ Unter Berücksichtigung der relativ geringen Akzeptanz und Zitationshäufigkeit von elektronischen Zeitschriften in den Fächern der klassischen Altertumswissenschaft, insbesondere in der deutschsprachigen Forschung ${ }^{7}$, stellt dieser Befund ein überraschend positives Zeugnis über die Stellung und positive Resonanz des noch jungen Projektes aus.

Seit Erscheinen der ersten Ausgabe im März 2006 verzeichnete die Zeitschrift innerhalb der ersten neun Monate ihres Bestehens knapp 10.500 Zugriffe. Bis 31. Dezember 2009 wurden insgesamt über 100.000 Seitenaufrufe für die Website www.fera-journal.eu und die mit ihr verbundenen Unterseiten registriert, was als Zeichen einer zunehmenden Bekanntheit gewertet werden darf. Die aufgrund ihres Charakters als fachwissenschaftliche Zeitschrift recht spezifischen Inhalte legen nahe, dass die Leserschaft überwiegend in der altertumswissenschaftlichen Fachwelt und zum Teil in angrenzenden Fächern (Geschichte, Humangeographie, Bibliothekswissenschaft, u. ä.) zu finden ist.

Die für den Zeitraum von März 2006 bis Dezember 2009 vorgenommene logfile-Analyse der Zugriffe auf die Website ergibt jedoch ein weitaus differenzierteres Bild über die Herkunft der Nutzer als ursprünglich angenommen.

\footnotetext{
${ }^{5}$ Zur Evaluierung von online-Fachveröffentlichungen in der digitalisierten Welt Cope - Kalantzis 2009.

${ }^{6}$ Neben der Auflistung in Bibliothekskatalogen umfasst dies auch die Erwähnung der Artikel in Literaturdatenbanken, wie etwa Gnomon online, oder den Bibliographischen Notizen der Byzantinischen Zeitschrift. Ferner werden Artikel aus FeRA sowohl in wissenschaftlichen onlinePublikationen aber auch herkömmlichen Printmedien zitiert.

${ }^{7}$ Samida 2006a, 1005-1006; Samida 2006b.
} 
Nur knapp 14 \% der Zugriffe auf die Startseite www.fera-journal.eu sowie die mit ihr verbundenen Unterseiten erfolgten über Universitäts- bzw. Bibliotheksnetzwerke. Im Rahmen der Auswertung wurden dazu die IP-Adressen nach den Schlagwörtern „uni“, „museum“, „ac.uk“ und „edu“ abgefragt. Diese Kombination erwies sich als eine gute Möglichkeit, um aus einer großen Datenmenge möglichst alle Zugriffe von einzelnen Universitäts- bzw. Bibliotheksrechnern aus dem In- und Ausland zu erfassen. Da von diesen wiederum nur einige wenige IP-Adressen aufgrund ihrer Adressenbestandteile, wie etwa „alt-gesch“, „klassarch“, „philologie“ u. ä., einem bestimmten Fach zugewiesen werden konnten, musste zwangsläufig auf eine weitere Unterscheidung nach Fachrichtungen verzichtet werden.

Die überwiegende Mehrheit der Zugriffe auf die Website erfolgte dementsprechend von IP-Adressen privater Internetprovider. Bei diesen ist verständlicherweise nicht ersichtlich, ob die betreffenden Nutzer einen Bezug zum Fach haben - also etwa Wissenschaftler oder Studenten sind, die über ihren privaten Internetzugang Fachtexte rezipieren - oder nicht. Interessante Deutungsmöglichkeiten über die Herkunft der Nutzer ergeben sich aus einer Betrachtung der Zugriffszeiten auf die Website: Unter Berücksichtigung der jeweiligen Zeitzonen für Europa und Nordamerika fällt auf, dass, wie nicht anders zu erwarten ist, über Universitätsserver überwiegend am Tag auf die Zeitschrift zugegriffen wird, während dies über private Internetanbieter in der Mehrheit außerhalb der regulären Arbeitszeit geschieht. Der Umstand, dass die Zeitschrift auch während regulärer Arbeitszeiten über private Internetanbieter aufgerufen wird, sowie die Zugriffe aus fachfremden Bereichen abzulesen an den IP-Adressen aus den Bereichen Medizin, Verwaltung und der Privatwirtschaft - und die Verweise auf FeRA-Artikel in Internetforen legen nahe, dass die „Frankfurter elektronische Rundschau zur Altertumskunde“ grundsätzlich auch von einer breiten fachfremden Leserschaft rezipiert wird. Wie die Zugriffe aus Ländern ohne eine besonders stark ausgeprägte Forschungstradition in der klassischen Altertumskunde (Südamerika, Süd- und Ostasien) zu deuten sind, ist zum gegenwärtigen Zeitpunkt noch unklar. ${ }^{8}$ Auch wenn der Anteil der fachfremden Leser nicht exakt zu quantifizieren ist, ist es dennoch bemerkenswert, dass außerhalb des Wissenschaftsbetriebes eine recht große Gruppe von Interessenten derart fachspezifischer Literatur existiert. Damit fügt sich der für die „Frankfurter elektronische Rundschau zur Altertumskunde“ ermittelte Befund in das generelle Bild eines hohen fachfremden Zugriffs ein, wie er bei anderen vergleichbaren altertumswissenschaftlichen Publikationsprojekten ebenfalls beobachtet wurde. ${ }^{9}$ Das allgemein erklärte Ziel der Open-Access-Bewegung ${ }^{10}$, nämlich Wissen und Information als allgemeine Güter durch einen freien Zugang nicht bloß ausgewählten Gruppen aus dem unmittelbaren institutionellen fachlichen Umfeld, sondern grundsätzlich allen Menschen zur Verfügung zu stellen, wird demnach erfüllt. Auch wenn durch das Projekt Wissen und Information außerhalb elitärer Strukturen weite Verbreitung findet, bleibt die ursprünglich intendierte Zielgruppe der Frankfurter elektronischen Rundschau zur Altertumskunde, hauptsächlich der wissenschaftliche

\footnotetext{
${ }^{8}$ Einen ähnlichen Befund bei den Princeton/Stanford Working Papers in Classic skizzieren Ober u. a. 2007, 232-233.

${ }^{9}$ Pritchard 2008, 7-8.

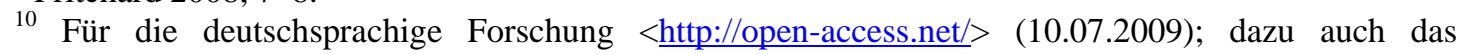
richtungsweisende Positionspapier der Deutschen Forschungsgemeinschaft (DFG) unter <http://www.dfg.de/forschungsfoerderung/wissenschaftliche_infrastruktur/lis/download/pos_papier_ele ktron_publizieren_0504.pdf $>$ (10.07.2009).
} 
Nachwuchs in den Fächern der klassischen Altertumskunde, proportional nur in geringerem Maße angesprochen.

Von ihrer Konzeption und ihrem Selbstverständnis her ist die „Frankfurter elektronische Rundschau zur Altertumskunde“ ganz auf den wissenschaftlichen Nachwuchs ausgerichtet, die Akzeptanz in dieser Zielgruppe fiel jedoch bislang geringer aus als erhofft. Im Gegensatz zu dem großen Interesse der Leserschaft war die Menge der eingereichten Manuskripte geringer als erwartet. Wie eingangs erwähnt stammen die meisten Beiträge von Autoren, die zum Zeitpunkt des Einreichens ihrer Manuskripte noch an der Dissertation arbeiteten, oder diese bereits vor Kurzem erfolgreich abgeschlossen hatten. Zudem ist zu beobachten, dass die Einsendung von Artikeln etwas abnahm, während gleichzeitig die Bereitschaft, Rezensionen zu verfassen, wuchs. In Gesprächen auf verschiedenen Doktoranden- bzw. PostGraduate-Veranstaltungen wurde von Kollegen wiederholt darauf verwiesen, dass das Interesse an einem solchen Projekt grundsätzlich sehr groß sei. Doch stelle der Zeitmangel ein großes Hindernis dar, tatsächlich Beiträge zu verfassen und einzureichen. Dies resultiert vermutlich aus der Zunahme zeitintensiver Verpflichtungen, denen Doktoranden während ihrer Promotion bzw. Promovierte nach ihrer Promotion vermehrt ausgesetzt sind: Dazu zählen die Übernahme von Lehrverpflichtungen, kürzere Abstände zwischen den Abgabeterminen einzelner Kapitel der Dissertation, die verpflichtende Aneignung von Schlüsselqualifikationen in der Promotionsphase und der zunehmende Druck aufgrund kürzerer Förderzeiten die Dissertation zielgerichtet und schnell zu Ende zu bringen.

Hinzu kommt, dass in den Fächern der klassischen Altertumskunde, wie auch in den Geisteswissenschaften insgesamt, die Bereitschaft zum elektronischen Publizieren noch nicht besonders ausgeprägt ist. Eine im Auftrag der Deutschen Forschungsgemeinschaft (DFG) durchgeführte und im Jahre 2005 publizierte Studie zum Publikations- und Rezeptionsverhalten unter besonderer Berücksichtigung von open access in verschiedenen Wissenschaftsbereichen zeigt auf, dass elektronisch publizierte Zeitschriften durchaus bekannt sind und genutzt werden. ${ }^{11}$ Zugleich macht diese Studie jedoch auch deutlich, dass gerade in den Geisteswissenschaften eine deutliche Zurückhaltung zu verzeichnen ist, als Autor eigene Beiträge in open access Zeitschriften zu publizieren. ${ }^{12}$ Auch im Fall der Frankfurter elektronischen Rundschau zur Altertumskunde zeigt sich, dass trotz des Modells einer elektronischen Zeitschrift ${ }^{13}$ mit institutioneller Verankerung (inkl. ISSN) und Qualitätsstandards wie bei herkömmlichen Print-Zeitschriften (Seitenzählung der Texte, Band- bzw. Jahrgangszählung der Ausgaben, Peer-Review-System mit eigenem Fachbeirat) der Reiz in einem elektronischen Medium zu publizieren in den Fächern der Altertumskunde immer noch sehr zögerlich wahrgenommen wird.

Unter diesen Rahmenbedingungen ist es auch nicht weiter verwunderlich, wenn Nachwuchswissenschaftler ihre wenigen freien Ressourcen genau abwiegen und eine Veröffentlichung ihrer Forschungsergebnisse aufgrund der größeren Reputation in gedruckten Medien bevorzugen. Für online-Zeitschriften bleiben dann meist Rezensionen oder kleinere Beiträge übrig. Auch die Möglichkeit, aus mehreren zugelassenen Publikationssprachen (im vorliegenden Fall deutsch, englisch und italienisch) zu wählen und Beiträge aus allen Teilfächern der klassischen Altertumskunde in einem Medium zu veröffentlichen, bringt keine merkbar positiven

\footnotetext{
${ }^{11}$ DFG-Bericht 2005, 22-52, bes. 39-45.

${ }^{12}$ DFG-Bericht 2005, 48-52.

${ }^{13}$ Zur Diskussion der unterschiedlichen Modelle Samida 2006a, 1012-1014.
} 
Auswirkungen mit sich. ${ }^{14}$ Zudem war es notwendig, auch bei der geringen Zahl der Beiträge auf die Qualität der Manuskripte zu achten, um die eigenen Standards zu erfüllen und dem Profil der Zeitschrift gerecht zu werden. Doch sind die Probleme, denen sich das FeRA-Projekt ausgesetzt sehen, kein singuläres Phänomen: Eine jüngste Studie für den angelsächsischen Raum bestätigt insgesamt die Tendenz einer zunehmend schwierigeren Situation im online-Publizieren von nachwuchswissenschaftlichen Forschungsergebnissen auf Zeitschriftenbasis. ${ }^{15}$

Zusammenfassend lässt sich festhalten, dass das Projekt einer von und für Nachwuchswissenschaftler organisierten lizenz- und kostenfrei abrufbaren onlineZeitschrift, die inhaltlich die ganze Breite der klassischen Altertumskunde umfasst, trotz der dargelegten Schwierigkeiten bislang relativ erfolgreich ist. Die Zeitschriftenbeiträge haben dank der online-Zugriffsmöglichkeit eine große Verbreitung sowohl innerhalb als auch außerhalb der Fachkreise gefunden. Die fachliche Rezeption der vermittelten Inhalte lässt sich rückblickend ebenfalls positiv bewerten. Dennoch ist es bedauerlich, dass gerade die jüngere Generation von Altertumswissenschaftlern aufgrund der skizzierten Umstände nicht mehr vom Einsatz elektronischer Medien profitiert. Dabei zeigen die Ergebnisse deutlich, dass mit online-Publizieren eine weitaus schnellere und bedeutend größere Verbreitung der eigenen Forschungsergebnisse erzielt werden kann, als durch die Publikation in herkömmlichen Printmedien. Dass immer mehr Institute von den Vorzügen elektronischen Publizierens überzeugt sind, ist an den Gründungen neuer elektronischer altertumskundlicher Zeitschriften abzulesen. ${ }^{16}$ Ein spezifisch von und auf den wissenschaftlichen Nachwuchs zugeschnittenes Konzept, wie dies bei der Frankfurter elektronischen Rundschau zur Altertumskunde der Fall ist, findet sich darunter jedoch nicht.

\section{Literatur}

Carver 2007

M. Carver, Archaeology Journals, Academics and Open Access, European Journal of Archaeology 10, 2007, 135-148.

Cope - Kalantzis 2009

B. Cope - M. Kalantzis, Signs of epistemic disruption: Transformations in the knowledge system of the academic journal, First Monday 14, 2009, $<$ http://firstmonday.org/htbin/cgiwrap/bin/ojs/index.php/fm/article/viewArticle

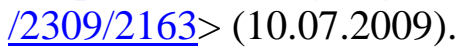

DFG-Bericht 2005

Publikationsstrategien im Wandel? Ergebnisse einer Umfrage zum Publikations- und Rezeptionsverhalten unter besonderer Berücksichtigung von Open Access, hrsg. von der Deutschen Forschungsgemeinschaft 2005,

\footnotetext{
${ }^{14}$ Dazu allgemein Carver 2007, 142-146.

${ }^{15}$ Salt 2007, 85.

16 Zuletzt die Gründung des Journal of Archaeology in the Low Countries im Mai 2009 als kooperatives Projekt mehrerer archäologischer Institute der Niederlande < $\underline{\text { http://www.jalc.nl/> }}$ (10.07.2009).
} 
Frankfurter elektronische Rundschau zur Altertumskunde 10 (2009)

$<$ http://www.dfg.de/dfg_im_profil/zahlen_und_fakten/statistisches_berichtswe sen/open_access/download/oa_ber_dt.pdf $>$ (10.07.2009).

Heath 2002

M. Heath, Editorial introduction, Leeds International Classical Studies 1.0, 2002, $\quad 1-8 \quad<$ http://www.leeds.ac.uk/classics/lics/2002/200200.pdf $>$ (10.07.2009).

Leiß 2006

C. Leiß, Elektronisches Publizieren im wissenschaftlichen Alltag. Überlegungen zur Integration elektronischer Publikationsformen in die Geisteswissenschaften, Bibliotheksdienst 40, 2006, 988-993.

Ober u. a. 2007

J. Ober - B. D. Shaw - W. Scheidel - D. Sanclemente, Toward Open Access in Ancient Studies. The Princeton-Stanford Working Papers in Classics, Hesperia 76, 2007, 229-242.

Pritchard 2008

D. Pritchard, Working Papers, Open Access, and Cyber-infrastructure in Classical Studies, Literary and Linguistic Computing 23, 2008, 149-162.

Salt 2007

A. Salt, Electric strata: Assemblage and changes in postgraduate publication on the internet, European Journal of Archaeology 10, 2007, 83-85.

Samida 2006a

St. Samida, Elektronische Zeitschriften in der Ur- und Frühgeschichtlichen Archäologie: Bestandsaufnahme und Analyse, Bibliotheksdienst 40, 2006, 1003-1012.

Samida 2006b

St. Samida, Prähistorische Archäologie: Von der 'Wissenschaft des Spatens' zur historischen Cyberwissenschaft?, zeitenblicke 5, 2006, <http://www.zeitenblicke.de/2006/3/Samida/dippArticle.pdf> (10.07.2009). 